\title{
The Effect of Anesthesia Method on Serum Level of Pro-Brain Natriuretic Peptide in Patients Undergoing Orthopedic Surgery
}

\author{
Alireza Mirkheshti ${ }^{1}$; Masoume Heidari Farzan ${ }^{1}$; Yashar Nasiri ${ }^{1}$; Kamran Mottaghi ${ }^{1}$; Ali \\ Dabbagh ${ }^{1,}$ \\ ${ }^{1}$ Anesthesiology Research Center, Shahid Beheshti University of Medical Sciences, Tehran, Iran \\ *Corresponding author: Ali Dabbagh, Anesthesiology Research Center, Shahid Beheshti University of Medical Sciences, Tehran, Iran. Tel: +98-9121972368, Fax: +98-2122074101, E-mail: \\ alidabbagh@sbmu.ac.ir
}

Received: May 30, 2014; Revised: August 8, 2014; Accepted: August 16, 2014

\begin{abstract}
Background: Surgical stress response is among the most severe stress tolerated by the patient, which needs suppression by anesthesia. Objectives: We assessed the effect of three methods of anesthesia on postoperative levels of pro-brain natriuretic peptide (pro-BNP) to determine the most effective one in preventing surgical stress response.

Patients and Methods: In a randomized clinical trial, 120 patients who were 18 to 65 years old and met inclusion and exclusion criteria were selected and randomly allocated to three groups of 40:Group A, general anesthesia plus epidural catheter; Group B, general anesthesia and intravenous patient-controlled analgesia; and Group C, spinal anesthesia plus intravenous patient-controlled analgesia. Results: There was no difference between three groups for basic characteristics and variables and baseline pro-BNP levels; however, postoperative pro-BNP levels in Groups A, B, and C were respectively63.8 $\pm 10.1,83.2 \pm 12.3$, and $51.5 \pm 8.5 \mathrm{ng} / \mathrm{L}$ (ANOVA, $\mathrm{P}=0.01$ ).

Conclusions: The results of the current study suggested that spinal anesthesia plus intravenous patient-controlled analgesia have the most favorable cardiac effects regarding postoperative levels of pro-BNP.
\end{abstract}

Keywords: Analgesia; Orthopedics; Brain Natriuretic Peptide

\section{Background}

Surgical stress response, including the intraoperative and postoperative acute pain, is among the most severe burden of stress tolerated by the patients. Anesthesia is used to suppress the stress response and make surgical manipulations more tolerable. Nonetheless, anesthesia could not be always performed perfectly to completely abolish the imposed surgical stress. In response to the imposed stress, the homeostatic status of the patients might change. Cardiovascular system is among the most important systems affected by homeostasis and braintypenatriuretic peptide (BNP) has been shown to be a good marker of myocardial injury $(1,2)$. In addition, BNP and N-terminal-pro-BNP have been shown to be useful prognostic tests in predicting the clinical outcome after a number of surgical operations (1-12). Higher levels of BNP and N-terminal-pro-BNP could detect high-risk patients who are undergoing operation for "major noncardiac surgery" regarding "cardiac mortality, all-cause mortality, and major adverse cardiovascular events" (9). Different anesthesia methods do not have the same effect on clinical outcome regarding their efficacy in suppressing this stress. A combination of the analgesic methods could be more effective while having fewer adverse effects of the anesthetics.

\section{Objectives}

In this study, we aimed to assess the effect of three anesthesia methods on postoperative levels of pro-BNP to determine which of these methods could prevent adverse effects of the surgical stress response.

\section{Patients and Methods}

In a randomized clinical trial, after considering the inclusion and exclusion criteria and after taking informed written consent, 120 patients were selected from all the patients entering the orthopedic operating room. The following conditions were considered as study inclusion criteria:

-Patients with 18 to 65 years of age

-Being operated by the same orthopedic surgeon

-Elective surgery

- Only lower limb operation

In addition, the following conditions were considered as exclusion criteria:

-History of underlying medical disorders

-History of drug or substance abuse

-Emergent or urgent surgery

-History of underlying low back pain

-Any decision by the patient to quit the study at any time during the perioperative period 
-Any technical failure that led to change the plan of study was conducted to exclude the case from the survey

The patients were randomly allocated to three groups of 40 based on a random table of numbers.

Group A: patients were anesthetized using general anesthesia (GA) for the surgical operation; moreover, an epidural catheter was inserted to administer epidural analgesia during the postoperative period. Therefore, they were the GA plus lumbar epidural analgesia (LEA) (GA + LEA) group.

Group B: Patients were anesthetized using GA for the surgical operation; in addition, intravenous patient-controlled analgesia (IVPCA) was used for suppressing acute postoperative pain (GA + IVPCA group).

Group C: Patients were anesthetized using spinal anesthesia for the surgical operation; in addition, IVPCA was used for suppressing acute postoperative pain (Spinal + IVPCA group).

The patients were anesthetized by a constant anesthesiologist. Moreover, all the patients were visited the night before the operation by a constant colleague to obtain written informed consent and description of the study. During any stages of the study, if any patients decided to leave the project, it was enough to tell one of the study colleagues. After patient entry to the operating room, each patient was visited by a constant colleague who had not been in touch with the patients the last night; moreover, he did not have any affairs regarding patient care in the postoperative period. Instead, another colleague outside the operating room visited the patients for postoperative care (including postoperative acute pain management). Inside the operating room, each patient was randomly allocated to one of the study groups. Selection for entering each group was done using a table of random numbers. GA in group A and $\mathrm{B}$ were done using the same method. In group $\mathrm{C}$, the patients received spinal anesthesia using a $25 \mathrm{G}$ spinal needle, through the L3 - L4 interspace and median approach. While monitoring the patients, they were sedated and then, changed to lateral decubitus position. Then the location was prepared and draped. A guardian nurse looked after the patient in this position. Then 3.5 $\mathrm{mL}$ of plain bupivacaine was administered after achieving positive result in barbottage test. At the end of the surgery, the patients were transferred to the post anesthesia care unit (PACU). The anesthesia block level was managed to be at about the level of T10. For induction of GA in groups A and B, after application of standard monitoring devices including electrocardiography, oxygen saturation, and noninvasive blood pressure monitoring, the patients received $10 \mathrm{~mL} / \mathrm{kg}$ of lactated Ringer's solution in 15 minutes. Then the patient received 0.02 $\mathrm{mg} / \mathrm{kg}$ of IV midazolam accompanied with $2 \mu \mathrm{g} / \mathrm{kg}$ fentanyl, $5 \mathrm{mg} / \mathrm{kg}$ of sodium thiopental, and $0.5 \mathrm{mg} / \mathrm{kg}$ of atracurium. Then they were intubated and receivedo.6\% to $1.2 \%$ isoflurane through inhalational route to keep Bispectral index (BIS) level in the range of 40 to 60 out of 100. In addition, neuromuscular monitoring was used to monitor administration of atracurium during operation and for reversing their effects at the end of surgery. For all the patients, tourniquet was applied. After termination of the operation, reversal of neuromuscular blockade and recovering full consciousness, the patients were extubated and transferred to the PACU. All surgeries lasted shorter than three hours. If duration of surgeries were longer than three hours, blood loss was more than $1 \mathrm{~L}$, or patients were hydrated more than 4 L during the operation, the cases were excluded from the study. In addition, any technical failure that led to changing the plan of study would exclude the case from the study. Preoperative plasma BNP levels were measured using the venous blood samples the night before operation; in addition, postoperative samples were taken 24 hours after surgery. For measurement of pro-BNP, we used the following method:

For preoperative plasma BNP measurement, we took a venous blood sample in a lithium-heparin tube and transferred them to a constant laboratory, using a box with room temperature. We had our patients to lie supine and calm for 15 - 20 minutes, without stress or agitation; since we wanted to eliminate confounding agents of plasma pro-BNP levels. Bayer ADVIA Centaur ${ }^{\mathrm{TM}}$ immunoassay operation (Bayer Healthcare LLC, Diagnostic division, Leverkusen, Germany) was our measurement method for plasma pro-BNP levels (13). Data entry and analysis was done using SPSS 16(SPSS Inc., Chicago, IL, USA). Data analysis was performed through ANOVA. P value $<0.05$ was considered statistically significant.

\section{Results}

There was no difference between the study groups regarding basic characteristics and variables including age, duration of surgery, and duration of recovery stay (Table 1). In addition, the three groups had no significant difference regarding baseline pro-BNP levels. Baseline and postoperative pro-BNP levels are demonstrated in Table 2.

Table 1. Preoperative Characteristics of the Patients in Study Groups $^{\text {a }}$

\begin{tabular}{lcccc}
\hline Variable & Spinal + IVPCA $^{\text {b }}$ & GA + IVPCA & GA + LEA $^{\text {b }}$ & PValue \\
\hline Age, $\mathbf{y r}$ & $25 \pm 6$ & $26 \pm 7.2$ & $24 \pm 4$ & $>0.05$ \\
Height, $\mathbf{~ c m}$ & $170 \pm 12$ & $168 \pm 14$ & $169 \pm 12$ & $>0.05$ \\
Weight, $\mathbf{~ k g}$ & $74 \pm 13$ & $74 \pm 12$ & $75 \pm 11$ & $>0.05$ \\
Gender & & & & $>0.05$ \\
Female & 5 & 4 & 3 & \\
Male & 35 & 36 & 37 & \\
\hline
\end{tabular}

a Data are presented as Mean \pm SD (Standard Deviation); each group consists of 40 patients.

b Abbreviations: IVPCA, intravenous patient-controlled analgesia; GA, general anesthesia; LEA, lumbar epidural analgesia; and ANOVA, analysis of variances. 
Mirkheshti A et al.

Table 2. Preoperative and Postoperative BNP Levels of the Study Groups $(n=40)^{a, b}$

\begin{tabular}{lcccc}
\hline Variable & Spinal + IVPCA & GA + IVPCA & GA + LEA & \multicolumn{1}{c}{ P Value for ANOVA } \\
\hline Preoperative BNP, ng/L & $27 \pm 6.2$ & $29 \pm 4$ & $28 \pm 5.7$ & $>0.05$ \\
Postoperative BNP, ng/L & $51.5 \pm 8.5$ & $83.2 \pm 12.3$ & $63.8 \pm 10.1$ & 0.01 \\
\hline
\end{tabular}

a Abbreviations: IVPCA, intravenous patient-controlled analgesia; GA, general anesthesia; LEA, lumbar epidural analgesia; ANOVA, analysis of variances; and BNP, brain-type natriuretic peptide.

${ }^{\mathrm{b}}$ Data are presented as Mean \pm SD (Standard Deviation); each group consists of 40 patients.

\section{Discussion}

The results of this study demonstrated that different anesthesia methods have different outcomes regarding the increase in postoperative levels of pro-BNP in patients undergoing orthopedic surgery. Patients receiving intraoperative spinal anesthesia accompanied with postoperative IVPCA had the least amount of increase in pro-BNP followed by GA plus LEA and GA plus IVPCA. These findings suggest that spinal anesthesia plus IVPCA was the most effective method in controlling surgical stress response. A systematic review and meta-analysis demonstrated that "elevated BNP or N-terminal-pro-BNP level" could be "a powerful predictor of mortality in septic patients" (11). Moreover, another study demonstrated that in patients admitted to intensive care unit, N-terminal-pro-BNP was elevated in correlation with the severity of illness and also, N-terminal-pro-BNP is an independent predictor of mortality" in critical care patients (4). Furthermore, in patients undergoing cardiac surgery, BNP could predict clinical outcome after surgery and is superior to traditional single marker approach (7). Moreover, in patients undergoing surgery of the abdominal aortic aneurysm, BNP levels had a strong correlation with postoperative cardiac events (10). Another study demonstrated that in patients undergoing emergent no cardiac surgery, adverse long-term clinical outcomes in patients at higher risk could be predicted when there was increased level of preoperative N-terminal-pro-BNP (5). Finally, another study demonstrated that in elderly patients undergoing surgery due to traumatic hip fracture, increased levels of preoperative BNP could discriminate major echocardiographic abnormalities (8). This final study had some common point with our study; however, there was no other study with similar methodology. Moreover, it could let us discriminate between the three methods regarding a sensitive marker for stress response.

\subsection{Study limitations}

There are a number of limitations to our study. The main limitation was a few pro-BNP checks in our study; in other words, we had one preoperative assessment and one postoperative assessment. More frequent assessments and calculating the area under curve for pro-BNP could have helped us to achieve more precise results. In addition, while the results show that the level of BNP in the spinal plus IVPCA was lower than other groups, the design of the study could not discriminate the effects of spinal anesthesia and postoperative IVPCA separately; the same limitation existed for postoperative epidural block. According to earlier studies, inflammatory markers could predict mortality in poor medical conditions such as in sepsis or after on-pump cardiac surgeries. However, it is not clear whether it could predict value work in less severe medical conditions such as an uncomplicated orthopedic surgery and whether we could translate our findings from less severe degrees of inflammation to better outcome in otherwise healthy candidates of surgery. Our current study does not demonstrate any specific finding regarding this issue and future studies should assess this hypothesis.

\section{Acknowledgements}

The authors would like to acknowledge the kind help of operating room physicians, nurses, and personnel regarding their assistance in handling the patients.

\section{References}

1. Lam LL, Cameron PA, Schneider HG, Abramson MJ, Muller C, Krum H. Meta-analysis: effect of B-type natriuretic peptide testing on clinical outcomes in patients with acute dyspnea in the emergency setting. Ann Intern Med. 2010;153(11):728-35.

2. Schneider HG, Lam L, Lokuge A, Krum H, Naughton MT, De Villiers Smit P, et al. B-type natriuretic peptide testing, clinical outcomes, and health services use in emergency department patients with dyspnea: a randomized trial. Ann Intern Med. 2009;150(6):365-71.

3. Rodseth RN, Padayachee L, Biccard BM. A meta-analysis of the utility of pre-operative brain natriuretic peptide in predicting early and intermediate-term mortality and major adverse cardiac events in vascular surgical patients. Anaesthesia. 2008;63(11):1226-33.

4. De Geer L, Fredrikson M, Oscarsson A. Amino-terminal pro-brain natriuretic peptide as a predictor of outcome in patients admitted to intensive care. A prospective observational study. Eur J Anaesthesiol.2012;29(6):275-9.

5. Farzi S, Stojakovic T, Marko T, Sankin C, Rehak P, Gumpert R, et al. Role of N-terminal pro B-type natriuretic peptide in identifying patients at high risk for adverse outcome after emergent noncardiac surgery. BrJ Anaesth. 2013;110(4):554-60.

6. Foroughi M, Rahimian H, Dabbagh A, Majidi M, Hekmat M, Beheshti $\mathrm{M}$, et al. Postoperative N-terminal pro-brain natriuretic peptide level in coronary artery bypass surgery with ventricular dysfunction after perioperative glucose-insulin-potassium treatment. J Cardiothorac Vasc Anesth. 2012;26(4):631-6.

7. Kallel S, Jmel W, Jarraya A, Abdenadher M, Frikha I, Karouia A. The role of procalcitonin and N-terminal pro-B-type natriuretic peptide in predicting outcome after cardiac surgery. Perfusion. 2012;27(6):504-11.

8. Pili-Floury S, Ginet M, Saunier L, Besch G, Bartholin F, Chopard 
$\mathrm{R}$, et al. Preoperative plasma B-type natriuretic peptide (BNP) identifies abnormal transthoracic echocardiography in elderly patients with traumatic hip fracture. Injury. 2012;43(6):811-6.

9. Ryding $\mathrm{AD}$, Kumar S, Worthington AM, Burgess D. Prognostic value of brain natriuretic peptide in noncardiac surgery: a metaanalysis. Anesthesiology. 2009;111(2):311-9.

10. Vetrugno L, Costa MG, Pompei L, Chiarandini P, Drigo D, Bassi F, et al. Prognostic power of pre-and postoperative B-type natriuretic peptide levels in patients undergoing abdominal aortic surgery. J Cardiothorac Vasc Anesth. 2012;26(4):637-42.

11. Wang F, Wu Y, Tang L, Zhu W, Chen F, Xu T, et al. Brain natriuretic peptide for prediction of mortality in patients with sepsis: a systematic review and meta-analysis. Crit Care. 2012;16(3):R74.

12. Watson CJ, Phelan D, Xu M, Collier P, Neary R, Smolenski A, et al. Mechanical stretch up-regulates the B-type natriuretic peptide system in human cardiac fibroblasts: a possible defense against transforming growth factor-beta mediated fibrosis. Fibrogenesis Tissue Repair. 2012;5(1):9.

13. Park JH, Shin GJ, Ryu JI, Pyun WB. Postoperative B-type natriuretic Peptide levels associated with prolonged hospitalization in hypertensive patients after non-cardiac surgery. Korean Circ J. 2012;42(8):521-7. 